University of Texas at El Paso

ScholarWorks@UTEP

\title{
$5-2017$
}

\section{Attraction-Repulsion Forces Between Biological Cells: A Theoretical Explanation of Empirical Formulas}

Olga Kosheleva

The University of Texas at El Paso, olgak@utep.edu

Martine Ceberio

The University of Texas at El Paso, mceberio@utep.edu

Vladik Kreinovich

The University of Texas at El Paso, vladik@utep.edu

Follow this and additional works at: https://scholarworks.utep.edu/cs_techrep

Part of the Biology Commons, and the Mathematics Commons

Comments:

Technical Report: UTEP-CS-17-43

To appear in Proceedings of the 10th International Workshop on Constraint Programming and Decision Making CoProd'2017, El Paso, Texas, November 3, 2017.

\section{Recommended Citation}

Kosheleva, Olga; Ceberio, Martine; and Kreinovich, Vladik, "Attraction-Repulsion Forces Between Biological Cells: A Theoretical Explanation of Empirical Formulas" (2017). Departmental Technical Reports (CS).

1124.

https://scholarworks.utep.edu/cs_techrep/1124

This Article is brought to you for free and open access by the Computer Science at ScholarWorks@UTEP. It has been accepted for inclusion in Departmental Technical Reports (CS) by an authorized administrator of ScholarWorks@UTEP. For more information, please contact Iweber@utep.edu. 


\title{
Attraction-Repulsion Forces Between Biological Cells: A Theoretical Explanation of Empirical Formulas
}

\author{
Olga Kosheleva, Martine Ceberio, and Vladik Kreinovich \\ University of Texas at El Paso, El Paso, TX 79968, USA \\ olgak@utep.edu, mceberio@utep.edu, vladik@utep.edu
}

\begin{abstract}
Biological calls attract and repulse each other: if they get too close to each other, they repulse, and if they get too far away from each other, they attract. There are empirical formulas that describe the dependence of the corresponding forces on the distance between the cells. In this paper, we provide a theoretical explanation for these empirical formulas.
\end{abstract}

\section{Formulation of the Problem}

Biological calls interact. Biological cells attract and repulse each other. For each type of cell, there is a certain distance $R_{1}$ at which there is no interaction.

- When the cells get closer to each other than this threshold distance, i.e., when the distance $r$ between the cells becomes smaller than $R_{1}$, then the cells repulse each other.

- On the other hand, if the two cells deviate further away that the threshold distance $R_{1}$, they start attracting each other.

As a result of these two forces, the cells stay at the same - biologically optimal - distance from each other.

Empirical formulas describing interaction between the cells. According to $[2,3,5]$, the interaction force $\mathbf{f}$ between the two cells at a distance $\mathbf{r}$ has the following form:

- when $r<R_{1}$, we have $\mathbf{f}=-k_{1} \cdot\left(\frac{1}{r}-\frac{1}{R_{1}}\right) \cdot \mathbf{e}$, where $r$ is the length of the vector $\mathbf{r}$ (i.e., the distance between the cells) and $\mathbf{e} \stackrel{\text { def }}{=} \frac{\mathbf{r}}{r}$ is the unit vector in the direction $\mathbf{r}$;

- when $r>R_{1}$, we have $\mathbf{f}=k_{2} \cdot\left(r-R_{1}\right) \cdot \mathbf{e}$.

Formulation of the problem. How can we explain these empirical formulas?

What we do in this paper. In this paper, we provide a theoretical explanation for the above empirical formulas. 


\section{Analysis of the Problem}

Qualitative requirements. We want to find the dependence $f(r)$ of the interactive force $f$ on the distance $r$ between the two cells.

To find such a dependence, let us consider natural requirements on $f(r)$.

Monotonicity. The larger the difference between the actual distance $r$ and the desired distance $R_{1}$, the larger should be the force. Thus:

- the repulsion force should increase when the distance $r$ decreases, while

- the attraction force should increase as the distance $r$ increases.

It should be mentioned that the empirical formulas satisfy this property - i.e., the corresponding dependencies $f(r)$ are monotonic.

Analyticity. This is a general phenomenon in physics, that all dependencies in fundamental physics are described by analytical functions, i.e., by functions which can be expanded in Taylor or, more generally, by Laurent series; see, e.g., [4]. For functions of one variable, this means that we must have

$$
f(r)=a_{0}+a_{1} \cdot r+a_{2} \cdot r^{2}+\ldots+a_{-1} \cdot r^{-1}+a_{-2} \cdot r^{-2}+\ldots
$$

In fundamental physics, this phenomenon is usually explained by the need to consider quantum effects: quantum analysis means extension to complex numbers - and analytical functions are, in effect, differential functions of complex variables; see, e.g., [4].

Again, it is worth mentioning that both empirical formulas - the formula corresponding to $r<R_{1}$ and the formula corresponding to $r>R_{1}$ - are analytical.

Tidal forces. The main objective of the forces between the two cells are to keep the cells at a certain distance. This motivates the direct effect of the forces:

- when the cells are two close, the repulsion force will make move apart, while

- when the cells are too far away from each other, the attraction force will make them get closer.

However, with this direct effect, there is also an undesired side effect, caused by the fact that cells are not points. As a result, different parts of the cell have slightly difference force acting on them. So, in addition to the overall force that makes the cell move in the desired direction, we also have tidal forces that make the parts of the cell move with respects to each other - i.e., make the cell compress or stretch.

In general, the tidal forces are proportional to the gradient of the force field (see, e.g., [4]), i.e., in this case, to the derivative $F(r) \stackrel{\text { def }}{=} \frac{d f}{d r}$.

From the biological viewpoint, tidal forces are undesirable, so they should be as small as possible. 
Scale invariance. Physical laws are formulated in terms of the numerical values of physical quantities. However, these numerical values depend on what measuring unit we select to describe this quantity.

For example, if we first measure distances in meters, and then start using centimeters instead, then all the numerical values get multiplied by a factor of 100. In particular, $2 \mathrm{~m}$ becomes $200 \mathrm{~cm}$.

In most fundamental physical laws, there is no physically preferred unit. In such situations, it make sense to require that the physical law not depend on the choice of the unit.

Of course it does not means that all the formulas remain unchanged if we simply change the measuring unit of one of the quantities. Usually, if we change the unit of one of the quantities, then we have to accordingly change the units of related quantities. However, after an appropriate re-scaling of all the units, all the formulas should remain the same.

In precise terms, scale-invariance of the dependence $b=B(a)$ between two quantities $a$ and $b$ means that for every $\lambda$, there exists a $\mu(\lambda)$ such that if we change $a$ to $a^{\prime}=\lambda \cdot a$ and $b$ to $b^{\prime}=\mu(\lambda) \cdot b$, the dependence remain the same: if $b=B(a)$, then we should have $b^{\prime}=B\left(a^{\prime}\right)$, i.e., $\mu(\lambda) \cdot b=B(\lambda \cdot a)$.

For the dependence of the force itself of the distance, there is clearly no scaleinvariance: indeed, in this case, there is a special distance $R_{1}$ at which the force is 0 . However, for the tidal force $F(r)$, interestingly, there is scale-invariance: namely, $F(r) \sim r^{-2}$ for small $r$ and $F(r)=$ const for large $r$; both are scaleinvariant formulas.

Now, we are ready to describe our result.

\section{Definitions and the Main Result}

The above properties take the following form:

\section{Definition 1.}

- By a force function, we mean a function $f(r)$ from positive numbers to real numbers.

- We say that a force function unction $f(r)$ is analyticaL if it can be expanded in Laurent series

$$
f(r)=a_{0}+a_{1} \cdot r+a_{2} \cdot r^{2}+\ldots+a_{-1} \cdot r^{-1}+a_{-2} \cdot r^{-2}+\ldots
$$

- We say that a force function is monotonic-at-0 if for sufficiently small $r$, its absolute value increases as $r$ decreases.

- We say that a force function is monotonic-at- $\infty$ if for sufficiently large $r$, its absolute value increases as $r$ increases.

- By a tidal force function corresponding to the force function $f(r)$, we mean its derivative $F(r)=\frac{d f}{d r}$.

- We say that a tidal force function is scale-invariant if for every $\lambda>0$, there exists $a \mu(\lambda)$ for which, for all $r$ and $a, a=F(r)$ implies that $\mu(\lambda) \cdot a=$ $F(\lambda \cdot r)$. 
Next, we should describe the property that the tidal forces should not grow too fast.

Definition 2. Let $f(r)$ be an analytical monotonic-at-0 force function $f(r)$, let $F(r)$ be its tidal force function, and let $F(r)$ be scale-invariant. We say that $F(r)$ grows fast if there exists another analytical monotonic-at-0 force function $g(r)$, with scale-invariant tidal force function $G(r)$, for which $\frac{F(r)}{G(r)} \rightarrow \infty$ as $r \rightarrow 0$.

Definition 3. Let $f(r)$ be an analytical monotonic-at- $\infty$ force function $f(r)$, let $F(r)$ be its tidal force function, and let $F(r)$ be scale-invariant. We say that $F(r)$ grows fast if there exists another analytical monotonic-at- $\infty$ force function $g(r)$, with scale-invariant tidal force function $G(r)$, for which $\frac{F(r)}{G(r)} \rightarrow \infty$ as $r \rightarrow 0$.

Proposition 1. Every analytical monotonic-at-0 force function $f(r)$ for which the tidal force function $F(r)$ is scale-invariant and does not grow fast, has the form $f(r)=\frac{c_{0}}{r}+c_{1}$ for some $c_{0}$ and $c_{1}$.

Proposition 2. Every analytical monotonic-at- $\infty$ force function $f(r)$ for which the tidal force function $F(r)$ is scale-invariant and does not grow fast, has the form $f(r)=c_{0} \cdot r+c_{1}$ for some $c_{0}$ and $c_{1}$.

Discussion. These are exactly the empirical formulas that we wanted to explain. Thus, we have a theoretical explanation for these formulas.

\section{Proofs}

$1^{\circ}$. Let us first see what we can conclude from scale-invariance of the tidal force function $F(r)$. By definition, this scale-invariance means that

$$
F(\lambda \cdot r)=\mu(\lambda) \cdot F(r)
$$

The function $F(r)$ is analytical, thus smooth. For smooth functions, every function $F(r)$ with this property has the form $F(r)=c \cdot r^{\alpha}$ for some constants $c$ and $\alpha$; see, e.g., [1].

This fact is easy to prove. Since the function $F(r)$ is smooth, the function $\mu(\lambda)$, which is equal to the ratio of two smooth functions $\mu(\lambda)=\frac{F(\lambda \cdot r)}{F(r)}$, is also smooth. Differentiating both sides of the equality $F(\lambda \cdot r)=\mu(\lambda) \cdot F(r)$ by $\lambda$ and taking $\lambda=1$, we conclude that $r \cdot \frac{d F}{d r}=\alpha \cdot F$, where $\left.\alpha \stackrel{\text { def }}{=} \frac{d \mu}{d \lambda}\right|_{\lambda=1}$.

By moving all the terms containing $r$ to one side and all the terms containing $F$ to another side, we conclude that $\frac{d F}{F}=\alpha \cdot \frac{d r}{r}$. Integrating both sides, we get $\ln (F(r))=\alpha \cdot \ln (r)+C$, for some integration constant $C$. Thus, for $F(r)=$ $\exp (\ln (F(r)))$, we get $F(r)=c \cdot r^{\alpha}$, where $c \stackrel{\text { def }}{=} \exp (C)$. 
$2^{\circ}$. Since the force function $f(r)$ is analytical, its derivative is also analytical. Thus, $\alpha$ should be an integer.

For $\alpha=-1$, integration of the above expression for $F(r)$ would lead to $f(r)=c \cdot \ln (r)$, which is not an analytical function. Thus, $\alpha \neq-1$, and integration of $F(r)$ leads to $f(r)=c_{0} \cdot r^{\alpha+1}+c_{1}$, where $c_{0} \stackrel{\text { def }}{=} \frac{c}{\alpha+1}$.

$3^{\circ}$. Monotonicity-at- 0 implies that $\alpha+1<0$, i.e., that that $\alpha+1 \leq-1$ and $\alpha \leq-2$. For $\alpha<-2$, we could take $g(r)=r^{-1}$ with $G(r)=-r^{-2}$ and thus, $\frac{F(r)}{G(r)} \sim \frac{r^{\alpha}}{r^{-2}}=r^{\alpha+2}$. From $\alpha<-2$, it follows that $\alpha+2<0$, hence $\frac{F(r)}{G(r)} \sim$ $r^{\alpha+2} \rightarrow \infty$ as $r \rightarrow 0$. So, all the cases when $\alpha<-2$ correspond to the tidal force function that grows fast. The only case when this function does not grow fast is the case $\alpha=-2$, which leads to $f(r)=c_{0} \cdot r^{-1}+c_{1}$.

$4^{\circ}$. Similarly, monotonicity-at- $\infty$ implies that $\alpha+1>0$, i.e., that that $\alpha+1 \geq 1$ and $\alpha \geq 0$.

For $\alpha>0$, we could take $g(r)=r$ with $G(r)=1$ and thus, $\frac{F(r)}{G(r)} \sim r^{\alpha}$. From $\alpha>0, r^{\alpha} \rightarrow \infty$ as $r \rightarrow \infty$. So, all the cases when $\alpha>0$ correspond to the tidal force function that grows fast. The only case when this function does not grow fast is the case $\alpha=0$, which leads to $f(r)=c_{0} \cdot r+c_{1}$.

The Propositions are proven.

Acknowledgments. This work was supported in part by NSF grants HRD0734825, HRD-1242122, and DUE-0926721, and by an award from Prudential Foundation.

The authors are thankful to Dr. Robert Smits from New Mexico State University for valuable discussions.

\section{References}

1. J. Aczél and H. Dhombres, Functional Equations in Several Variables, Cambridge University Press, Cambridge, UK, 1989.

2. E. Di Costanzo, A. Giacomello, E. Messina, R. Natalini, G. Pontrelli, F. Rossi, R. Smits, and M. Twarogowska, "A discrete in continuous mathematical model of cardiac progenitor cells formation and growth as spheroid clusters (cardiospheres)", Mathematical Medicine and Biology, 2017.

3. M. R. D'Orsogna, Y. L. Chuang, A. L. Bertozzi, and L. S. Chayes, "Self-propelled particles with soft-core interactions: patterns, stability, and collapse", Physical Review Letters, 2016, Vol. 96, pp. 104302-104305.

4. R. P. Feynman, R. B. Leighton, and M. L. Sands, The Feynman Lectures on Physics, Addison-Wesley, Redwood City, California, 2005.

5. J. Joie, Y. Lei, M.-C. Durrieu, T. Colin, C. Poignard, and O. Saut, "Migration and orientation of endothelial cells on micropatterned polymers: a simple model based on classical mechanics", Discrete and Continuous Dynamical Systems. Series B, 2015, Vol. 20, pp. 1059-1076. 